\title{
Adaptation of Simulated Annealing for Rate of Penetration Optimization in Automated Drilling
}

Zachary Cox

Follow this and additional works at: https://researchrepository.wvu.edu/etd

\section{Recommended Citation}

Cox, Zachary, "Adaptation of Simulated Annealing for Rate of Penetration Optimization in Automated Drilling" (2017). Graduate Theses, Dissertations, and Problem Reports. 5405.

https://researchrepository.wvu.edu/etd/5405

This Thesis is protected by copyright and/or related rights. It has been brought to you by the The Research Repository @ WVU with permission from the rights-holder(s). You are free to use this Thesis in any way that is permitted by the copyright and related rights legislation that applies to your use. For other uses you must obtain permission from the rights-holder(s) directly, unless additional rights are indicated by a Creative Commons license in the record and/ or on the work itself. This Thesis has been accepted for inclusion in WVU Graduate Theses, Dissertations, and Problem Reports collection by an authorized administrator of The Research Repository @ WVU. For more information, please contact researchrepository@mail.wvu.edu. 
Adaptation of Simulated Annealing for Rate of Penetration Optimization in Automated Drilling

\title{
Zachary Cox
}

Thesis submitted to the Statler College of Engineering and Mineral Resources at West Virginia University in partial fulfillment of the requirements

for the degree of

Master of Science

in

Petroleum and Natural Gas Engineering

\author{
H. Ilkin Bilgesu, Ph.D., Chair \\ Mark Sindelar, Ph.D. \\ Samuel Ameri M.S.
}

Department of Petroleum and Natural Gas Engineering

Morgantown, West Virginia

2017

Keywords: Drilling Automation, Simulated Annealing, Artificial Intelligence, Algorithm Adaptation

Copyright 2017, Zachary Cox 


\section{Adaptation of Simulated Annealing for Rate of Penetration Optimization in Automated Drilling Zachary Cox}

Drilling automation has focused on developing predictive controls based on existing formation and well sites for which abundant data is available. These methods are not suitable in new locations where there is little information and where drilling data has not been recorded. This study focuses on a proof-of-concept to allow drilling in locations with little or no data available by determining drilling parameters via an artificial intelligence algorithm. The methods used were tested for use in the second annual Drillbotics competition sponsored by the Drilling Systems Automation Technical Section (DSATS) of the Society of Petroleum Engineers (SPE).

This study addresses the difficulties and challenges faced in adapting artificial intelligence optimization algorithms for use with real-world applications. Furthermore, the limitations of such a system are examined and the breakdown between the algorithm and operational limitations are explored. A review of past drilling automation attempts and research was conducted and existing problems identified.

This research was completed on a pilot-scale drilling rig used by West Virginia University in the Drillbotics competition. The rig was used with multiple samples made in-house in order to provide a variety of materials, inclinations, and drilling conditions. The review of the test was subject to professional judges to provide an unbiased decision and served to advance this study. 


\section{Content}

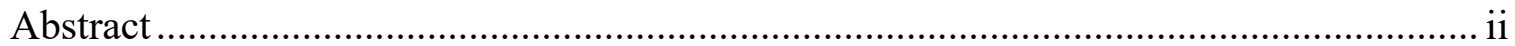

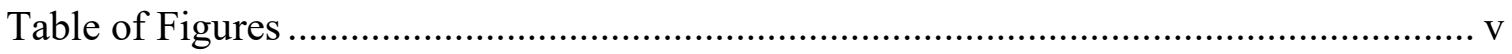

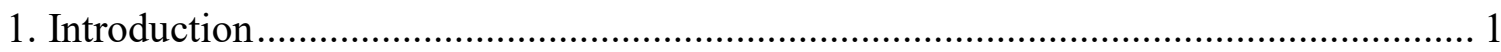

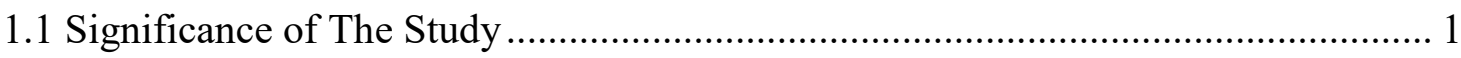

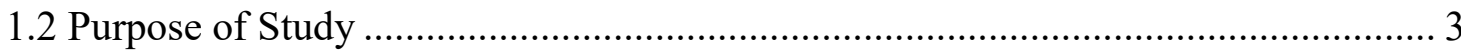

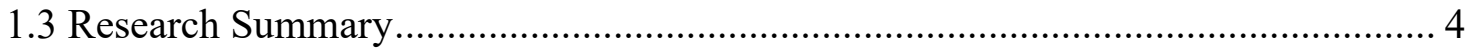

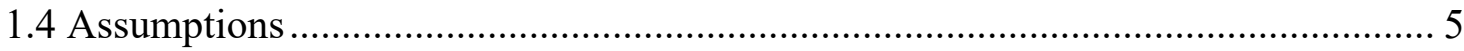

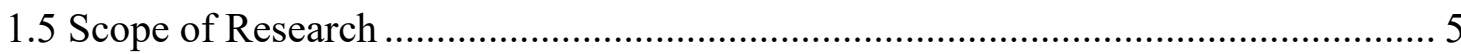

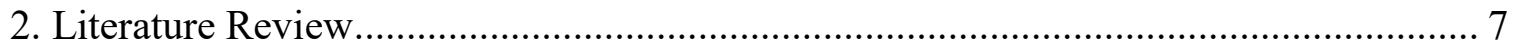

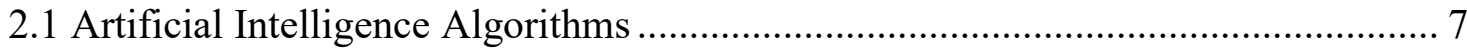

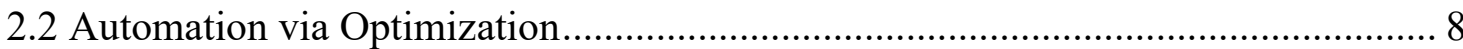

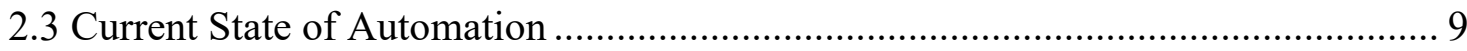

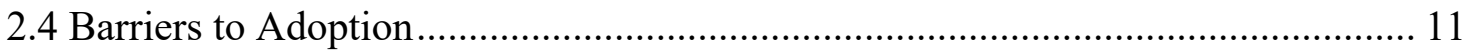

3. Design of Small-Scale Drilling Rig Control System .................................................. 12

3.1 Design Considerations and Constraints........................................................... 12

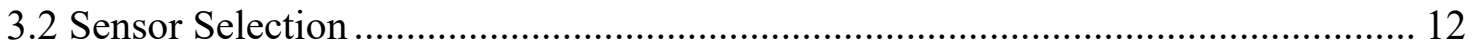

3.3 Control and Data Acquisition Hardware .............................................................. 13

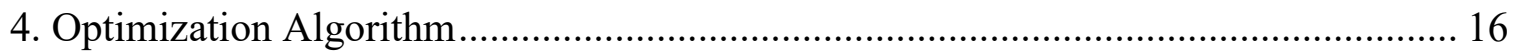

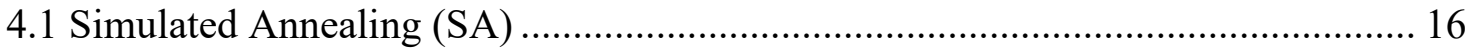


4.2 Algorithm Adaptation

4.3 Advantages of Optimized Parameters .................................................................... 21

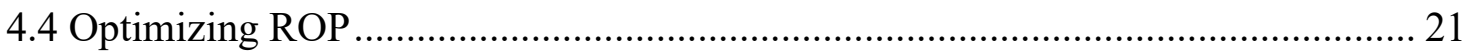

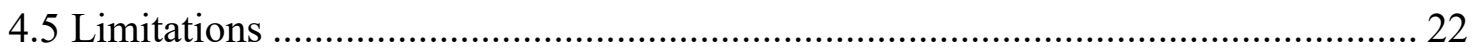

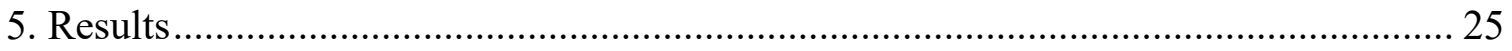

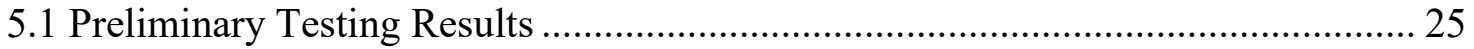

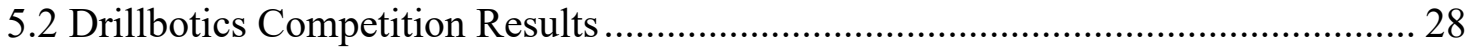

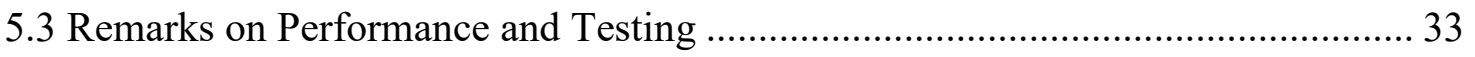

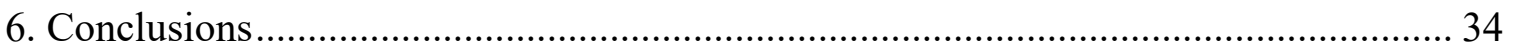

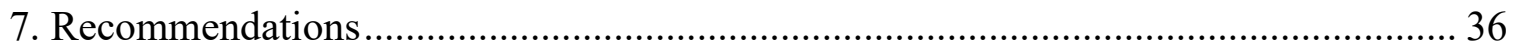

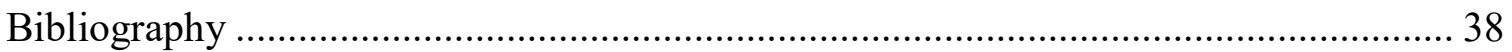




\section{Table of Figures}

Figure 1 Control Architecture ................................................................................... 15

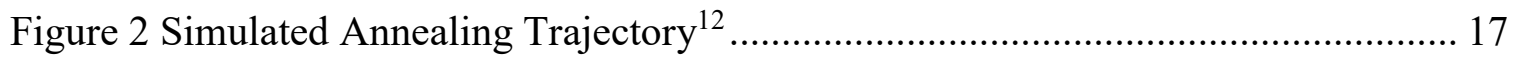

Figure 3 Simulated Annealing Flowchart * ANFIS: adaptive neuro-fuzzy inference

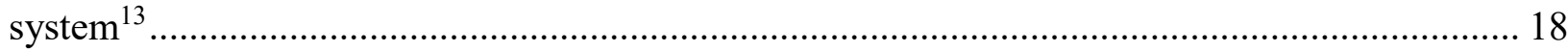

Figure 4 Simulated Annealing Output ……………………....................................... 20

Figure 5 Simplified Simulated Annealing Method Used................................................ 20

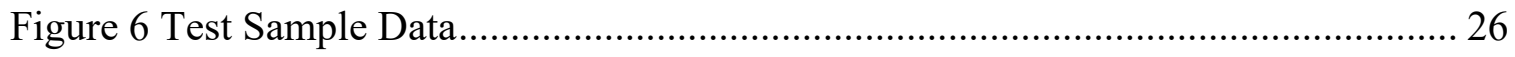

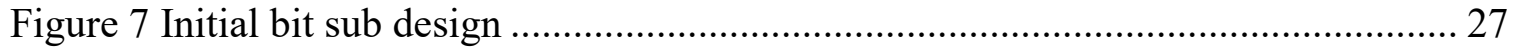

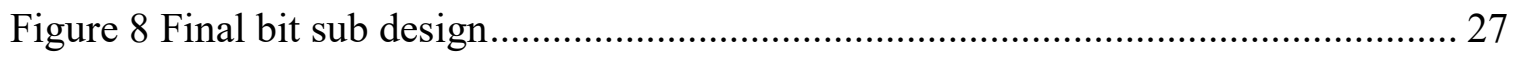

Figure 9 Competition Results …………………………........................................ 30

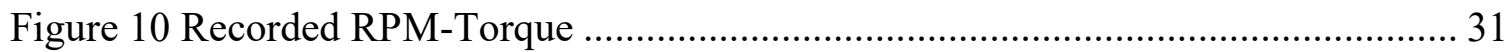

Figure 11 Recorded System Power Consumption ........................................................ 32 


\section{Introduction}

\subsection{Significance of The Study}

The Oil and Gas industry is entering an automation revolution, as new technology provides accurate and fast downhole data and control processes are refined to provide safe, reliable, and efficient drilling operations. The original surge in unconventional gas reservoirs, gas deposits which require hydraulic fracturing to produce, was characterized by rising demand and large profit margins despite the inefficiencies present at most drilling operations. As the industry faces a continuing supply glut, these inefficiencies are beginning to disappear, with alternatives providing higher operating margins. Unlike many industries that have already become largely automated, the drilling process faces multiple difficulties with this transition, specifically the number of separate companies involved in a single drilling process. However, as collaboration within the industry increases and new standards are brought forth for automated processes, the adoption of new drilling systems with augmented control is set to become the new standard.

The forces driving automation are similar to those in other industries, namely safety, increased efficiency, and ultimately lower costs. In terms of heightened safety, an automated rig allows for less workers to be present, eliminates the need for workers in the most hazardous areas, and continuously self-monitors to defeat unsafe or threatening conditions. Some of these benefits can be seen in the systems that already exist, such as automated movement of drill pipe, which removes roustabouts from one of the more traditionally dangerous areas on the rig. Self-monitoring rigs are also able to determine failure conditions or track potentially dangerous data directly from the downhole sensors, removing human error and the delay in time for a worker to notice the 
change in conditions. This in turn leads to less incidents and could improve worker safety while also increasing returns in the oil and gas industry.

Efficiency is the prime reason most industries begin to research automation, particularly when faced with difficult economic prospects. Part of the difficulty in automating a process like drilling is the number of unknowns and the vast differences between wells, even those within the same formation. Therefore, a single standardized procedure would be inefficient, cause bit damage, fluid loss, and suboptimal drilling speeds. This highlights the need for automation that can actively change and adapt to downhole conditions. A common practice with typical wells in well-known areas is to model the system prior to drilling to develop the plans necessary for an efficient drill path. However, this varies widely based on the experience and abilities of the crew and is still subject to human error and unknown variances in the system. A controlled system reduces non-productive time and maintains an optimal rate of penetration without the risk of damage to equipment or personnel.

Lastly, the appeal of automation lies in the long-term cost savings such a system is likely to offer. This is due to the savings from increased safety and efficiency as well as the natural effects of automation, such as reduction of the necessary workforce. As well complexity increases and demographics shift the overall experience of the workforce, automated systems can actively adapt to new conditions regardless of the controller, allowing for maximum yields even after the exodus of tenured employees. This is due to the lower level of knowledge needed to oversee such a process as opposed to actively controlling it.

Furthermore, it should be noted that due to the nature of the drilling process, it will likely never be fully automated, as the system will rely heavily on human-computer interface. Finally, 
while automation is clearly poised to become a big factor in the industry, it is only as a part of the process where drillers and engineers are still needed to plan and respond to any situation.

\subsection{Purpose of Study}

This study focuses on the use of artificial intelligence optimization, that is simulated annealing (SA), to allow for adaptive, automated drilling on a small-scale rig. The results of this study were used for the second annual Drillbotics competition sponsored by the Drilling Systems Automation Technical Section (DSATS) of the Society of Petroleum Engineers (SPE). This study

considers the possible options and algorithms for automation, and proposes optimization algorithms for unknown formations. Primarily this study looks at the difficulties and challenges faced when using computer algorithms for drilling processes. Alternatively, this study challenges methods which have been used in previous research or actively in the field.

The intention of this study is to provide a proof of concept for the adaptation of computer algorithms for automated drilling. As drilling automation moves into active operations and continuing academic research is conducted, a wider range of options will emerge, stemming from this and similar studies. A literature review of drilling automation was conducted and the problems identified. An automated protocol was developed, and drilling algorithm refined to serve as framework for future projects focused on automation.

This research was conducted on the small-scale drilling rig designed by West Virginia University DSATS team for the second annual Drillbotics competition. The rig was used for several tests prior to this. However, the competition provided the unique opportunity for a completely unknown testing sample to be used. The protocol was compared by an impartial panel 
of judges against other modern protocols used by different universities. As such, the competition served to advance this study and provide unbiased review from a selection of professionals.

\subsection{Research Summary}

This research examined the requirements to adapt artificial intelligence optimization techniques to select real-time drilling parameters, such as weight on bit (WOB), rotational velocity (RPM), and mud flow rate (gpm). This application is not limited by industry, rather it is a technique that can be further applied to open-ended, real world problems. This includes drilling applications for wells in addition to scientific sample collection regardless of the media. Due to the slow pace of automation adoption in the oil and gas industry this research aims to provide another method to encourage adaptation of new drilling methods. Currently, most drilling automation has been overly dependent on accurate models used to predict the drilling parameters needed. This does not allow for any new locations to be drilled as it requires information from previous drilling. The data may not be accurate and may have inaccuracies that are difficult to pinpoint depending on the age of the dataset.

Newer, faster, and more accurate sensors have enabled automation to a certain extent by providing the necessary data for an algorithm to be reliably executed. However, the methods used are not independent and are reliant on old data. This is not ideal, and a better option would be one which is reactive to the drilling environment. Thus, adapting an optimization algorithm to control drilling parameters in real time can be applied for drilling unknown formations. In turn, the process could be simulated and perfected without physical runs. This decreases the cost of tuning and reduces mechanical wear and fatigue on the components. 
Once ready, the system can be run on the small-scale rig and interfaced with additional hardware to provide feedback and additional information to the user. The system uses an accurate sensor assortment which is calibrated using certified instruments to ensure proper measurements are recorded. Secondary equipment, while unnecessary for the rig to operate, allows for simple data transfer and real-time updates via a wired connection. The system operates via standard start and stop protocols with drilling being fully controlled by the algorithm.

\subsection{Assumptions}

This study is restricted to small scale drilling unit and the adaptability of this application to be used in full-scale operations require further investigation. No attempt is made to recreate accurate field conditions or further adapt the setup to resemble active operations. It is assumed that all methods and data collected can be used in a similar setup by use of real time communications. This is paramount to the operation of the drilling algorithm and a prerequisite for using the method described. It is anticipated that the approach used in this study can easily be extended to other drilling systems where additional data are available as inputs to the algorithm developed in this study.

\subsection{Scope of Research}

The algorithm and control coding was developed in the Arduino coding environment and a hardware limited version of $\mathrm{C}++$ was used to control the equipment in a setup similar to a programmable logic controller. This allows the equipment to be completely changed and any programming to be accomplished with the resources available for Arduino compatible 
microcontrollers (boards). A combination of boards was used to distribute command and control of the various systems and sensors, while also recording data and streaming results in real time. The boards used to control the motors utilized a basic feedback loop to control individual drilling parameters. Simulated annealing was used to determine the drilling parameters needed; this data was then ported to the control boards. The operation of the rig is entirely optimization based and no categorization or prediction is performed in the program.

The small-scale rig constructed for the research is restricted to drilling vertically and relies on the optimization algorithm to reduce vibration, produce a vertical drill path, and minimize bit wear. Along string sensors, sensing devices that are mounted on the drill string or in the drilling assembly, as well as motor sensors and control feedback were used to update the drilling data and adapt when a new rock type was encountered. Testing was conducted using many rock types and samples, as well as laboratory tested homogeneous sandstone samples. The optimization process was evaluated for the different samples and formation types to determine the degree of success in maximizing Rate of Penetration (ROP) and time required to drill. 


\section{Literature Review}

\subsection{Artificial Intelligence Algorithms}

Artificial Intelligence (AI) algorithms are largely considered by the general public to consist of machines that interpret some form of data (speech, environment, object movement) and respond in the correct manner. Recently, this has largely been present in speech recognition, digital voice assistance through in home devices and cell phones. However, artificial intelligence deals with a broad set of problems with results that prove useful both practically and academically. These algorithms are responsible not only for model prediction and laboratory calculations, but also power optimized travel routes for individuals and industry. ${ }^{1}$

The commonality present in all $\mathrm{AI}$ is that the main goal falls into one of two categories: classification or optimization. This statement is generally held to be true for single use algorithms. That is, if multiple algorithms are used together then the resultant may not fall within one of these two categories. However, for the purpose of this research, the two-category classification is sufficient as deep classifications are of little relevance to this pilot study.

To identify the candidates to be used for any AI implementation it is first necessary to determine which of the two categories one must use. Classification protocols are useful for operations such as image searching, where the program can be trained to pick the proper picture given a text input. Alternatively, optimization can be used to determine the highest or lowest value possible for a given problem, such as the shortest route possible or the maximum accrued profit. This clarification points out the need for properly classifying the problem and determining if the solution needed is predictive or reactive. 
AI which is predictive in nature must be created with some knowledge of the conditions present. This is often present in the form of machine learning, where the algorithm adopts parameters from a test set, and verifies the results. Naturally, this is the common type of classification used. Reactive protocols are those that use data which is immediately available. This is more often present in optimization where the program simply takes a starting point and finds a good solution. Thus, if there is a large amount of information available, a predictive classification protocol is the best option. Alternatively, if there is little existing information, a reactive optimization protocol would better serve the user.

For AI to be properly utilized, the problem must be significantly complex, otherwise the solution will be deterministic despite the algorithm used. There are two important factors to consider: is the problem too simple, or is the absolute best possible solution necessary? In the case of a trivial solution an exhaustive search will provide the global optimum solution whereas AI will provide a probabilistic solution. If, however, the model is too complex, a solution which is near optimal must be considered as the global deterministic solution may be too resource intensive to find.

\subsection{Automation via Optimization}

The purpose the Drillbotics competition, and real world operations, is to drill quickly and accurately without sacrificing system integrity. The approach discussed here varies from many of the real-world automation studies performed as they rely primarily on modeling and predictive analysis of the formation to be drilled. An approach via optimization as opposed to prediction or categorization provides an overabundance of benefits: ${ }^{2}$ 
- Optimizing ROP minimizes vibration, bit wear, and energy required

- Any formation can be drilled without extensive study

- Less time is used to trip in and out for bit replacement

- Adaptation to unforeseen or poorly modeled events

- Ability to change drilling parameters based on real time data

- Characterization of formation types based on the optimized parameters

A categorization approach would require the testing of multiple conditions prior to any use to build a catalog of rock types. While this could be a better approach in theory, it is not feasible to test every type of formation considering many different properties that can be encountered during drilling. In a similar way, prediction would appear to be a superior choice as well. However, it too is completely dependent on the quality of the data available and is useless for a wildcat well, which is drilled in an unexplored area. PID control is often mentioned in control systems as well, however this is only acceptable for maintaining set point parameters of individual systems. PID control is not sufficient to adapt in response to incoming data, it must be used along with a program which updates set points in response to the data. These control systems are used to support the automation procedure but do not constitute a feasible solution for full control and automation.

\subsection{Current State of Automation}

Adoption of automated protocols in the drilling industry has been slow when compared to other areas primarily due to three factors: economic cycles, safety/regulatory concerns, and complexity of the system. Economic cycles tend to vary between boom and bust, where little research is done during the downturn and boom cycles ignore new technology due to the lost time 
needed to adapt newer tools. This, in turn, leads to the abandonment of new technology in the bust cycle as there is little funding available to support new advancements. However, the research fueled by higher operating margins actively benefits the companies during the downturn as they can apply the most economical solutions to remain profitable.

Concerns over safety come largely from those skeptical of automation, believing incorrectly that a human can better interpret and handle the data than a computer. This also leads to the problem of regulation, typically requiring the experience of an operation until the verification of a new system and possibly afterwards. The extreme conditions present in drilling operation do provide ample opportunity for a system to fail and measures must be taken to ensure an automated system will not result in undue downtime or injury.

System complexity is a major driver in the automation of the oil industry as the process of drilling a well is more intensive than that of machine construction/assembly or even autopilot in aircraft or ships. To understand how these processes can be so simply automated it is necessary to look at the most advanced sector of automation, primarily manufacturing. Here the unknowns have been eliminated. For example, the material that is feed into the machines is known as are its dimensions. The process never needs to change or adapt and the parts are all the same. The most difficult areas to address are inconsistency in the materials used, tool wear, and equipment calibration as the machines will continue to execute the same tasks in the same manner as before without correction. Drilling, on the other hand, will always have a certain number of unknowns, and the process is complicated. Whereas manufacturing has one machine for each section, drilling relies on one rig which must simultaneously monitor drilling as well as the surrounding strata and easily switch between the multiple operations that are necessary from a single rig. 
Automated systems are also dependent on the quality of the data they receive and the traditionally slow transfer speeds that are associated with downhole tools. While there are new solutions for this old problem, such as wired drill pipe, most companies aren't willing to accept the additional cost. This is true for the down hole sensors as well, which are often corrected after retrieval based on laboratory results and known formation corrections. , $^{3,5}$

\subsection{Barriers to Adoption}

Automation has made strides in the oil and gas industry. However, it has mostly been seen in noncritical functions. One example is the automatic loading of drilling pipe, which has greatly improved safety on the rigs as it eliminates one of the single most dangerous positions. However, this fails to compare to the complexity of automating the drilling procedure itself. Some automated procedures have been successful. These are often predictive in nature, and take place in areas that have been previously explored. Thus far, automated rigs have seen limited success without any companies perfecting the technology or widespread adoption by a supermajor oil company. ${ }^{6}$

The barriers that still exist are primarily twofold: lack of reliable, fast, and accurate downhole data and the lack of research towards both reactive and predictive algorithms to safely drill. The former is being addressed by several companies which are primarily working to increase the speed of data being transmitted form downhole sensors. The latter has seen little progress with most attempts being made using predictive methods to semi-automate drilling in well-known areas. However, each of these is needed to provide a truly autonomous drilling station which will be adaptable in any location. ${ }^{7}$ 


\section{Design of Small-Scale Drilling Rig Control System}

\subsection{Design Considerations and Constraints}

As the rig used for this study was designed for the Drillbotics competition, primary constraints were dictated by the limitations imposed by the competition guidelines. These defined the size, power, available budget, and bore size. The only drilling parameter constrained by the guidelines was weight on bit which was to be capped at $20 \mathrm{lbs}$. maximum. Other constraints were inherent with components used. The drilling motor, as one example, had a maximum RPM of 1750 with a minimum for drilling set in the code. The components similarly had limited flow rate, as was the data rate used by the control boards. These constraints are necessitated by the overall design of the rig, and would require more attention on rigs that are more capable.

\subsection{Sensor Selection}

The single most important factor in sensor selection was the relation to real application parameter that they monitor. This includes the weight on bit, rotary speed, mud flow rate, mud pipe pressure, depth penetrated, and power consumed. The rig proportions necessitated the use of miniaturized sensors which could be mounted to get readings as close to the point at which they are experienced. Moreover, the sensors must be able to communicate with the data acquisition hardware with low latency. Owing to the budget restrictions of the competition, price primarily dictated sensor selection. This led to the use of proportional cost, allowing for prominent sensors to be allotted a larger portion of the budget than those which needed lower resolution or were ancillary. 
The rig utilized a total of eight primary sensors which fed raw data to the slave nodes upon request. These sensors were used to measure WOB, RPM, gpm, pipe pressure, drill string torque, axial loading, vertical loading, and electric power consumption. These sensors communicated with the same slave nodes that controlled the components that they were intended to measure. The quantities measured are read by the slave nodes, converted into actual readings to be sent out by the master node to be saved or transmitted further to a display for the operator.

Triaxial strain gauges were mounted directly on the drill string and connected to the slave nodes via a multiple connector slip-ring. This allowed for a direct measurement of torque and had the added benefit of providing a backup source for the weight on bit measurement. Of notable exception, the weight on bit sensor was mounted on the drill motor mount as it could not be located with the bit. This caused some interference by the vibrations as it provided an indirect measure of weight on bit, after accounting for the load of the entire drill sting and the bit sub. This was calibrated prior to every run to minimize the error involved in an indirect measurement.

\subsection{Control and Data Acquisition Hardware}

The industrial standard for automation is the Programmable Logic Controller (PLC), an easy to use, robust piece of hardware that is simple to program and intended to run industrial processes reliably. Unfortunately, the budget used did not allow for the purchase of a PLC and all necessary components. As such the components used were utilized in a way that mimicked the operation of a PLC, and allowed for similar functionality albeit with significantly heavier

overhead. This was accomplished with microcontroller boards setup in a master/slave configuration. A typical PLC works as the master, additional inputs and output cards act as slaves 
receiving commands and sending back data that is requested. Ordinarily the PLC itself handles all the data, the input/outputs are simply additional sources of control and measurement. ${ }^{8}$

For this setup, the master is an Intel Edison System on a Chip, essentially a miniature two core compute-modules that can be programmed to work with the Arduino controllers. ${ }^{9}$ Four Arduino Uno controllers are used as slaves, input/output acting as the data acquisition hardware and simultaneously controlling the components. The master issues commands and handles the algorithm requesting data from the slaves as needed and issuing new commands as the program develops new solutions. ${ }^{10}$ This setup, shown in Figure 1, eliminates latency in the algorithm that can be caused by interrupts in the I/O or the delay of sensors when data is requested. Furthermore, operations that require a continuous signal, such as motor rpm, do not affect the master node. A simplified plot of this setup is shown below, the master node is the only one capable of sending and receiving data without a prompt. As such all instructions come via the master and are sent out to the slaves to the components. Data requested is picked up by the slaves and transmitted when requested by the master. 


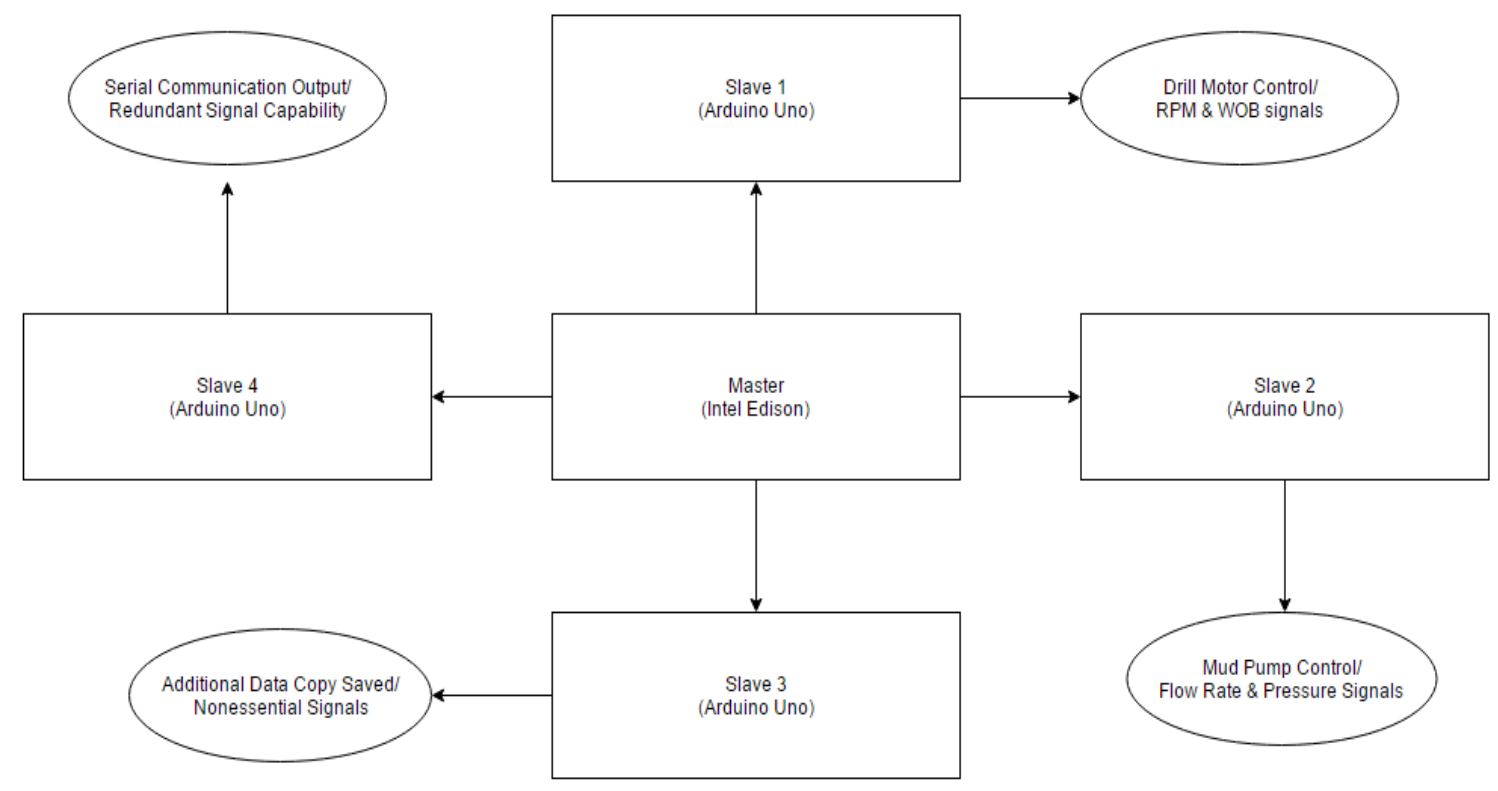

Figure 1 Control Architecture

This configuration allows for each node to be programmed independently and, if necessary, hot swapped should a single node malfunction. This ultimately allows for only the master to be updated once the slaves have received initial programing as they only react to instructions from the master. Data is sent to the master where it is formatted and transmitted for transmission for viewing or for a redundant copy to be saved. The master node always saves a raw copy of the drilling data as it is collected. 


\section{Optimization Algorithm}

\subsection{Simulated Annealing (SA)}

Simulated Annealing is an optimization technique developed analogous to the annealing of metals as the atoms cool and create a more ordered structure. The technique itself is a metaheuristic used in certain computer science applications where simple hill climb type algorithms are insufficient as there may be multiple local minima and maxima. To use simulated annealing a starting temperature must be defined, this is a relation to the uncertain nature of the system initially. A high starting temperature takes longer to "cool" or become ordered while a low temperature may not allow enough time to find a best solution. The process uses random jumps to explore the range of the parameters and allows for a relatively small number of samples to be used to find a local $\mathrm{max} / \mathrm{min}$. While there is no guarantee that the solution found will ever be the global optimum, it does find a probabilistic solution. As different points are tested they are constantly compared to the previous solutions and if they are better, there is the possibility that the area of the last solution is abandoned for the new solution. This can also happen if the solution is worse as the algorithm is seeking out multiple areas of the parameter range. However, this is up to a random generation and the better the new solution the more likely it will be selected, more so as the system "cools". The ideal progression of the solution is shown below in Figure 2., ${ }^{8,11}$ 


\section{Simulated Annealing}

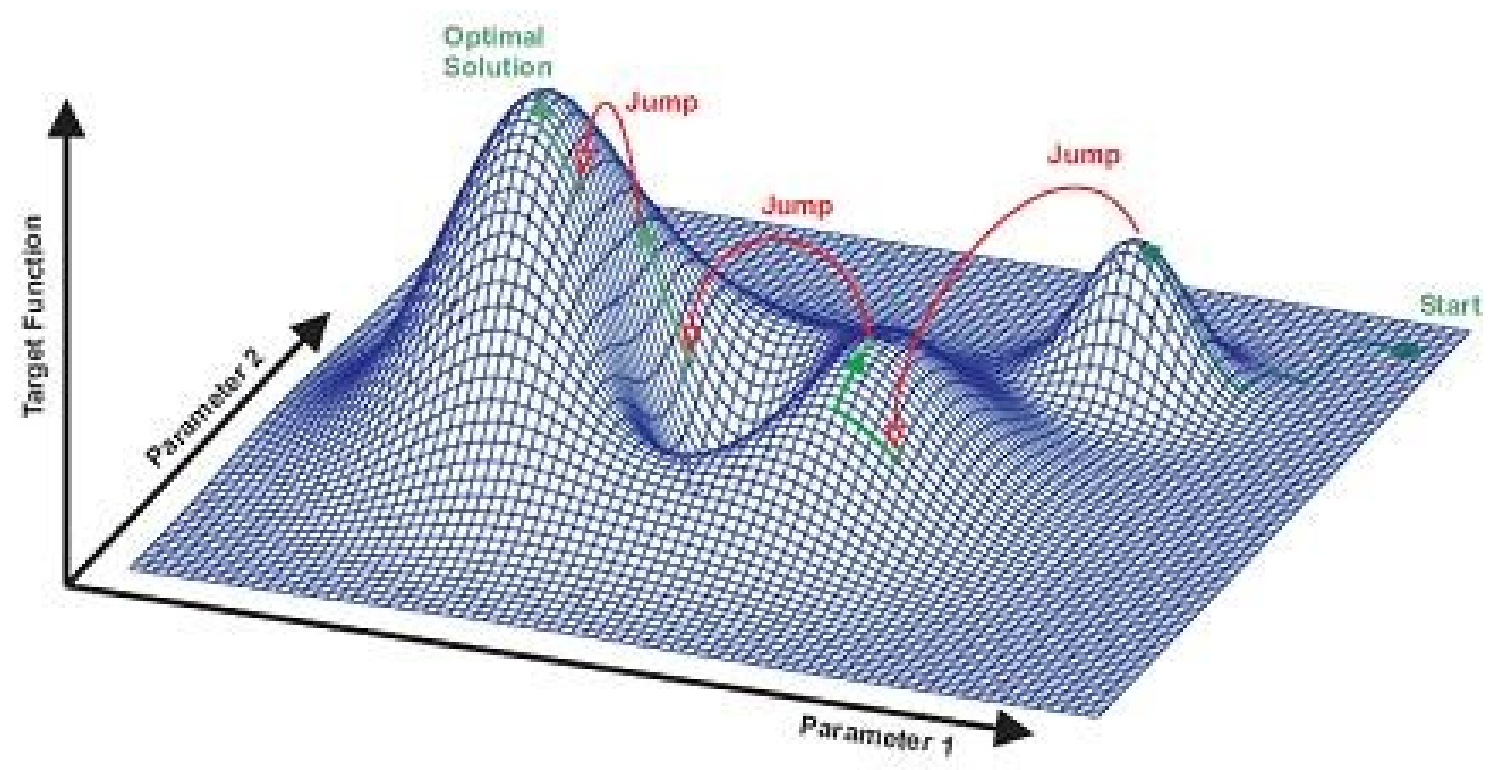

Figure 2 Simulated Annealing Trajectory ${ }^{12}$

The jumps shown are the result of a new solution being accepted as optimal. This is less likely to happen as the system cools. After each new set of parameters is evaluated the system cools. The algorithm can be limited in the number of combinations that are run, or the temperature can be used as the limiting factor to end the run. In either case, the criteria for termination must be set or else the program can run indefinitely. The typical flow for an unmodified program is shown in Figure 3. This is the basis for all SA programs and allow alterations to suit a specific application. This example terminates once a certain temperature is reached. 


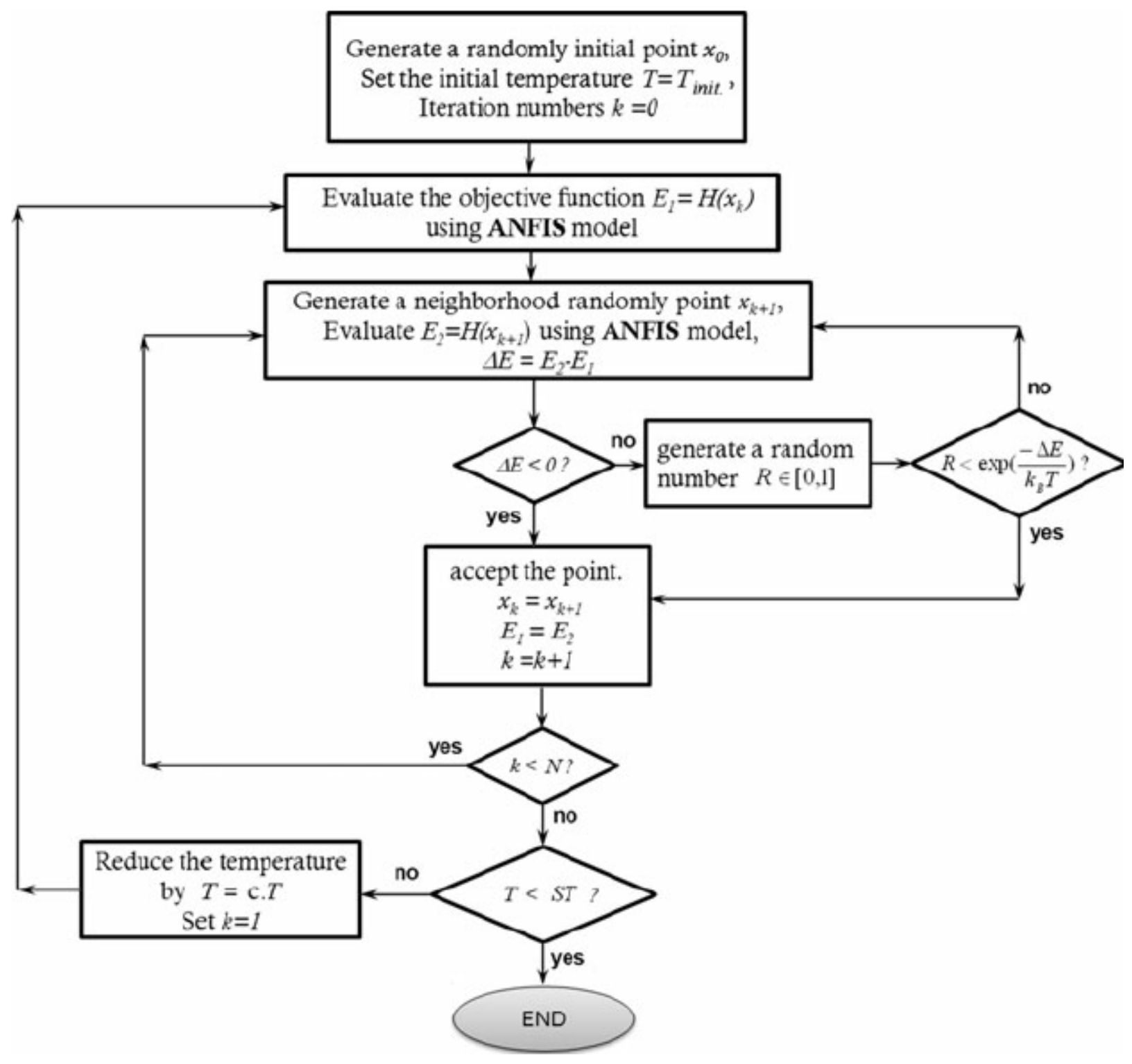

Figure 3 Simulated Annealing Flowchart* ANFIS: adaptive neuro-fuzzy inference system $^{13}$ 


\subsection{Algorithm Adaptation}

To make use of the algorithm, changes must be made so that it can accommodate the two limiting factors of the drilling process: the latency inherent in the application of new parameters and the low number of sample sets that can be used to find some maxima. The former is due to the time the new parameters must be able to run to provide reliable data back to the program. The latter is due to a tradeoff between the time needed to retrieve more data and the number of data points that provide a "good enough" answer. These factors are determined through experimentation, both within the program and in practice. The discretization of the variables reduced the issue as it decreased the total number of samples that were possible. Once the number of reasonable samples and the latency needed for accurate data were determine the algorithm could be altered to its final form.

This was insufficient however, as the cooling schedule needed to be tuned to the specific problem. To make proper use of the number of samples being used, the program had to be further altered to cool at a rate that caused the solution to converge prior to termination. If the temperature of the system stayed high for the entire run, the results would be random. If the system cooled too quickly the results would resemble the original parameters that were chosen to start the protocol. The system was simulated to determine an optimal cooling rate without the need for physical runs. The result is shown below in Figure 4 and a flowchart of the final protocol is shown in Figure 5. Note that in Figure 4 below the program can run many sample points. This is to illustrate the benefit of reducing the total number of samples to find a probabilistic solution. 


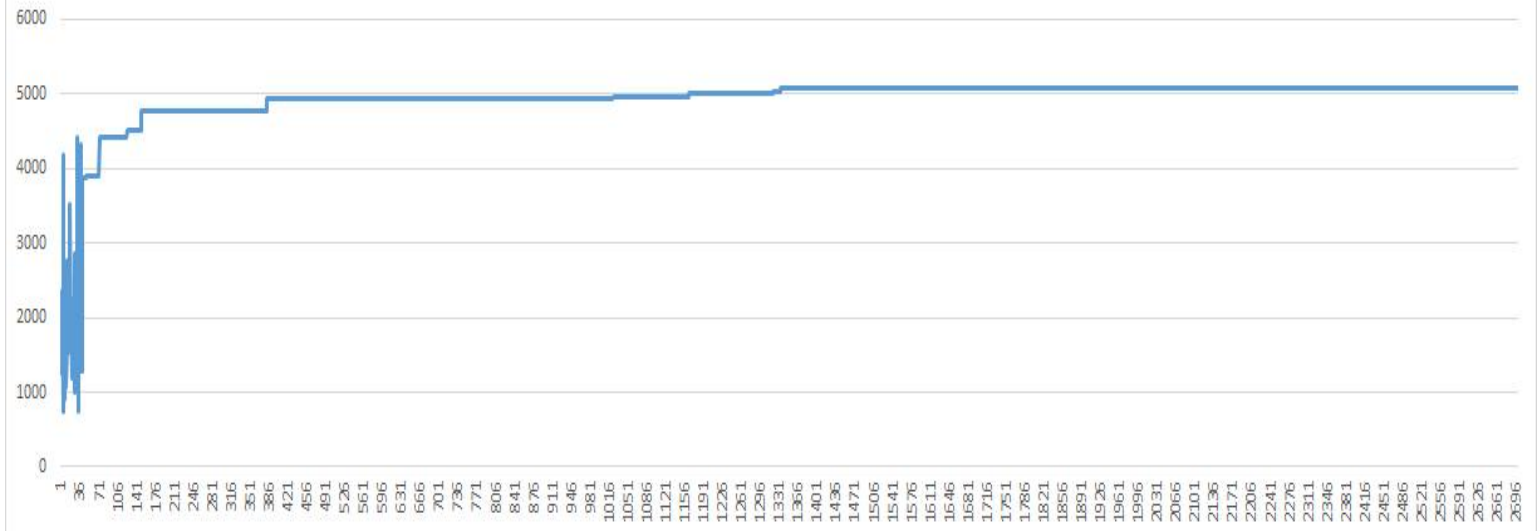

Figure 4 Simulated Annealing Output

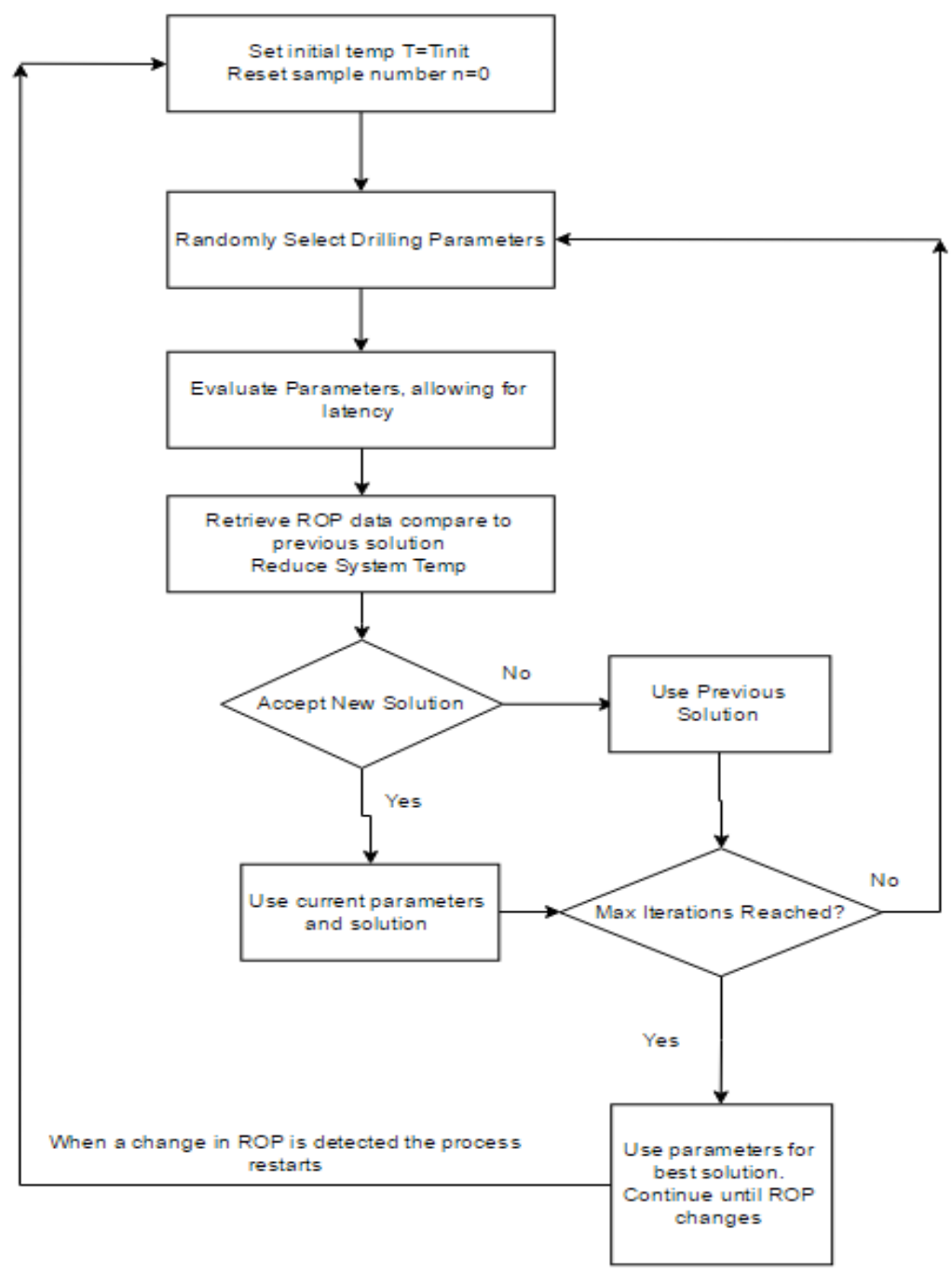

Figure 5 Simplified Simulated Annealing Method Used 


\subsection{Advantages of Optimized Parameters}

The clear advantage of optimizing the individual parameters is inherent in the dependency of the system. Namely, since the individual values are interdependent, any attempt to optimize a single parameter and then calculate the others would likely fail. This is due in part to the reactive nature of the algorithm since the calculated values could not be based off the performance caused by the newly selected parameter. This also creates a problem that is difficult enough to justify the use of AI, if the system were simple enough to use only one optimized parameter an exhaustive search method would serve adequately.

Furthermore, the parameters are all variables needed to calculate the rate of penetration (ROP). This means that the algorithm can calculate theoretical ROP and compare it with the measured ROP allowing for comparative analysis. This gives the system a feasible goal to achieve and a way to determine which solution is best. It also allows for the simulation to be run to analyze the performance of the program prior to implementation.

\subsection{Optimizing ROP}

In order for any protocol to optimize a solution, it is necessary to prescribe a basis to rate the provided solutions. This can be done many ways and can take into account any number of parameters provided they have been weighted or used in a formula which delivers a numerical output that can be used. In this specific approach, there are several choices that could be used such as, time/ft., mechanical specific energy, rate of penetration, vibration, or total energy consumption. This list can be expanded by combinations of the former variables or use of any arbitrary factor 
present in the system. It would be completely possible to rank the optimization of the process by water used per distance penetrated per time.

The choice was made to use ROP only as the standard of ranking due to its importance to the cost of drilling time and the secondary effects associated with optimizing ROP. To understand the benefit, it is useful to consider the effect of vibration on the system. Vibration is, in effect, lost energy that is removed from the process and here provides no useful input. Thus, if ROP is optimal then vibration is minimal. Verticality of the well bore is affected in much the same way, as ROP (as measured in this setup) is only calculated for vertical depth, so that optimal ROP will produce the most vertical shaft. This in turn will improve mechanical specific energy (MSE) as there is less wasted energy, and will decrease the time per foot parameter as well as improve total energy consumption. ROP is the only variable that can improve each of these parameters as it is improved. ${ }^{14,15}$

It is important to note that the enhanced ROP value does not necessarily optimize these other parameters. Rather it provided a feasible standard for the program to be tested with. This and the secondary effects of optimizing ROP justified the use of it as the single parameter to optimize.

\subsection{Limitations}

The algorithm is limited by several distinct parts of the process, the inherent latency, physical limitations of components, and the discretization of parameters. The individual components each provide their own limitations in terms of operating environment and range of operation. Much of this is mitigated in the installation and housing of components and can largely 
be ignored. Other issues may originate with the use of multiple components and interference between devices.

The components which place limitations on the algorithm are those which are controlled either by sensor capabilities or their own operating range. These are the lift motor (WOB), the drill motor (RPM), and the pump (gpm). The range of units used must be within the operating range of these component which limits the field of variables that can be chosen. This does have the side effect of allowing for fewer tests to determine a reasonable optimum. This hardware limited parameter range allows for a quicker search of the available values, meaning less searching for an optimized answer.

The discretization of parameters is typical for automated procedures and can be considered in most industries and applications. However, due to the limitations of the hardware and software used, the discretization is a significant issue. In order to quickly and easily send a single variable signal between the Arduinos, an eight-bit value must be used. This, in turn, leads to all operating values to exist on a scale of 0 to 255 . While this is not a problem for the WOB (accuracy of $\sim 0.098$ lbs.) it does present potential issues with the drill and pump motors (RPM accuracy of $\sim 7 \mathrm{rpm}$ ). However, these ranges are well within an acceptable range for this project as they are all less than $1 \%$ of the total range, and are of little concern at this scale. Should the process be adapted to a larger scale, the value would have to increase as well.

The inherent latency of the system is the single most problematic drawback that must be considered. Normally, an optimization algorithm works instantly, without having to wait any appreciable time for the output of a single operation. However, in this setup the program must wait for the parameters to change and the physical operation to set to the new parameter before the drilling can be measured for every data point. The resulting problem is twofold: there must be 
enough time between testing to allow for the parameters to update and the new drilling data to be read and the latency must be considered with the number of samples taken to reduce the total amount of time needed for each drill off test.

Another factor to consider is the storage used for parameters within the discrete range of the programming language employed. Despite the fact that modern controllers offer large ranges, regularly up to 64 bits, the system utilized here is limited to 8 bit (256 discrete values). In practice, this means the system could have a maximum search space of $256^{3}(167,77,216)$ possible selections. However, since the full range of senor detection is not possible to use and limits are built into the software, the actual search space used had 62,500 selections which was used to tune the algorithm. 


\section{Results}

\subsection{Preliminary Testing Results}

Preliminary testing was performed with a multilayer sample that was prepared in house. The sample was approximately 14 in. deep and was comprised of rock, cement, foam, and mineral layers in various inclinations. The top of the sample was coated with an epoxy resin to ensure a smooth, level interface between the sample and the bit. The depth/time graph below shows six samples that were all aligned near the center of the sample to compare similar results. While the actual depth at which materials were encountered is not exact in each test, the orientation and thickness at when they were contacted are similar. The results of these tests are shown in Figure 6. 


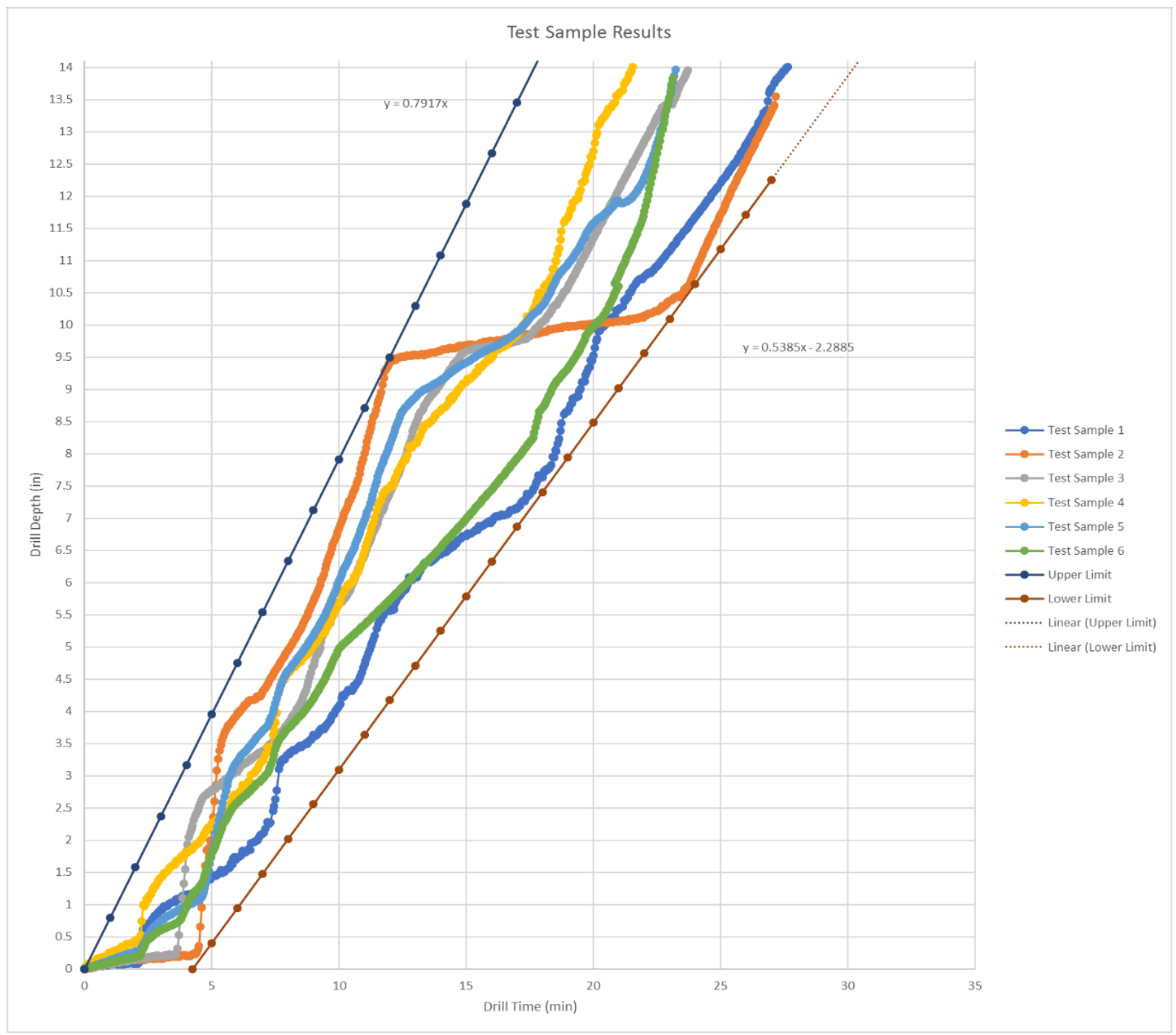

Figure 6 Test Sample Data

It should be noted that Test Sample 2 became stuck at a depth of 9.5 in at a time of 12 min., and because of this, the algorithm sensed a change in ROP and re-optimized the parameters. While this did result in the drill pipe freeing itself, the low ROP remained as the set point. This is because a low ROP is easy to maintain, and until the program can sense a change it will not try to reoptimize. This was corrected for by redesigning the bit sub to ensure a smooth transition by including more stabilizers. The original bit sub design can be seen in Figure 7 and the revised version in Figure 8. 


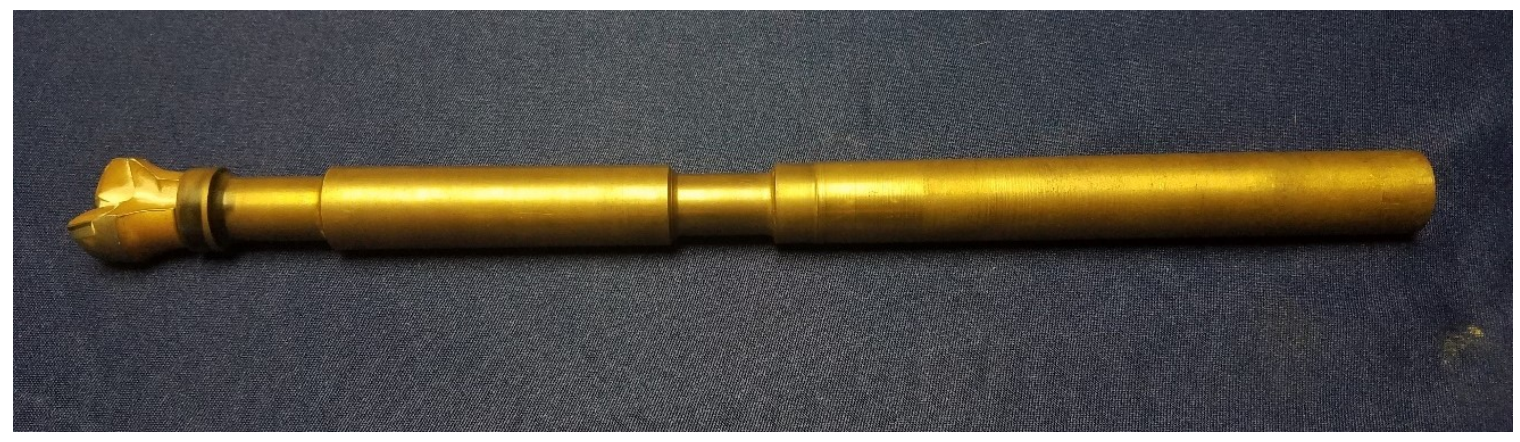

Figure 7 Initial bit sub design

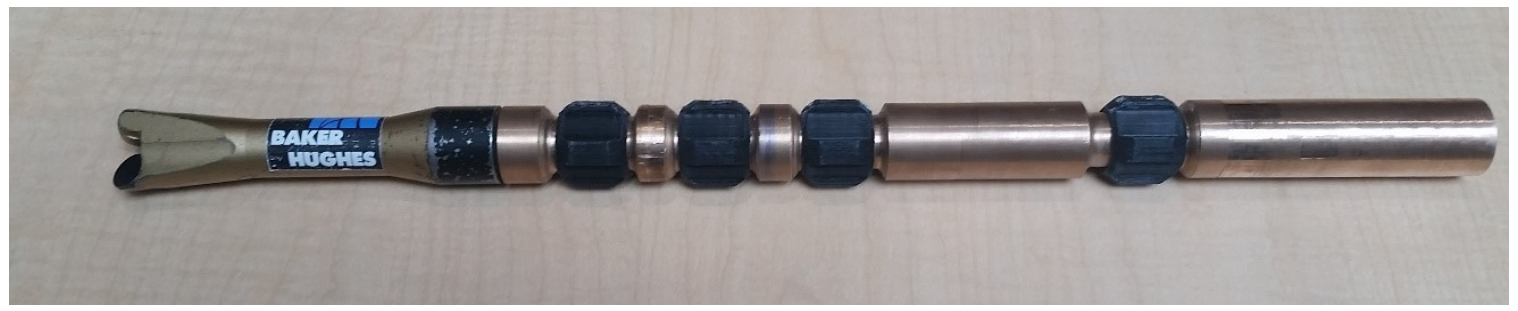

\section{Figure 8 Final bit sub design}

The results show that the algorithm worked as intended, but they also exhibit one of the issues that arise when the algorithm is adapted for real-life applications. The time required to drill the test sample varied from 21.5 to 27.7 minutes, a difference of 6.2 minutes. Taking the upper and lower bounds by using the outlier points available, the slope of the upper bound is higher than that of the lower bound. As shown in Figure 6 this leads to the interpretation that with the limitations imposed on the number of samples used to determine an optimum solution, the final result will vary proportionally to the complexity of the sample.

Here the depth of the sample is part of the complexity involved, and so as the depth increases and different layers are encountered the possibility of each optimal solution being similar decreases. This is expected behavior, and the algorithm is tuned for the sample size to be tested. However, it does show that as the system becomes more complex, the ability to predict the outcome decreases. 
Further analysis of the graph showed that different tests do have very similar slopes at similar depths. This is an example of the optimization being similar for the strata in different runs. A clear example of this is shown for the first three minutes in every test where there is a layer of resin used to level the top of the sample and allow for a good seal between it and the bell nipple used. The drill bit had difficulty penetrating the smooth glass-like surface, so the algorithm tended to select slow rotation with higher weight on bit. The slow progress caused the nearly flat beginning seen in each dataset.

These results are due to the program being tuned with the latency of the system, and the requirement to wait long enough for the drilling process to create useful data. The data sets would look more similar if more iterations were used for simulated annealing. However, at some point, this begins to negatively affect the amount of time required as the latency for each test begins to outweigh the time saved by a slightly better ROP. The diminishing returns seen require a specifically adapted algorithm to capitalize on the balance for a unique application. Nonetheless, the preliminary testing provided average ROP which was significantly higher than that reported from other rigs in the competition, and similar to the highest ROP achieved by manual control.

\subsection{Drillbotics Competition Results}

The opportunity to compete against other universities to drill an unknown sample was used to support the use of AI controlled drilling. Part of this includes the operation of the system in full view of competition judges, open to unbiased questions and challenges of the system in operation. During this testing, no operational issues occurred that could be attributed to systematic failure. However, components provided by the committee for the test did fail, notably the drill string 
connections. These failures caused the drill string to spin independently of the drill bit. While this hindered the drilling operation, it did show the active adaptation of the algorithm to continue drilling. When the failure occurred, the program picked up the change in ROP and adjusted the parameters in an attempt to continue drilling. This led to moderate success as the RPM dropped and maximum WOB was applied to force the bit to turn despite the loss of a secure mechanical connection. The connection failed in subsequent trips downhole and each failure is marked in Figure 9. In addition to the coupling failure, it was discovered that the test sample included void spaces which also create a spike in the ROP.

One drawback to the mounting of the strain gauge used to measure the WOB was the interference caused by vibrations induced while drilling. While the actual WOB was calibrated and tested multiple times the vibrations caused the unfiltered data to pick up the vibrations in the support chain. This caused WOB readings to vary wildly between zero and maximum despite the weight applied. This can be seen in Figure 9 and was compensated for by using a moving average to show the maintained weight that was used and adjusted for by the program. 


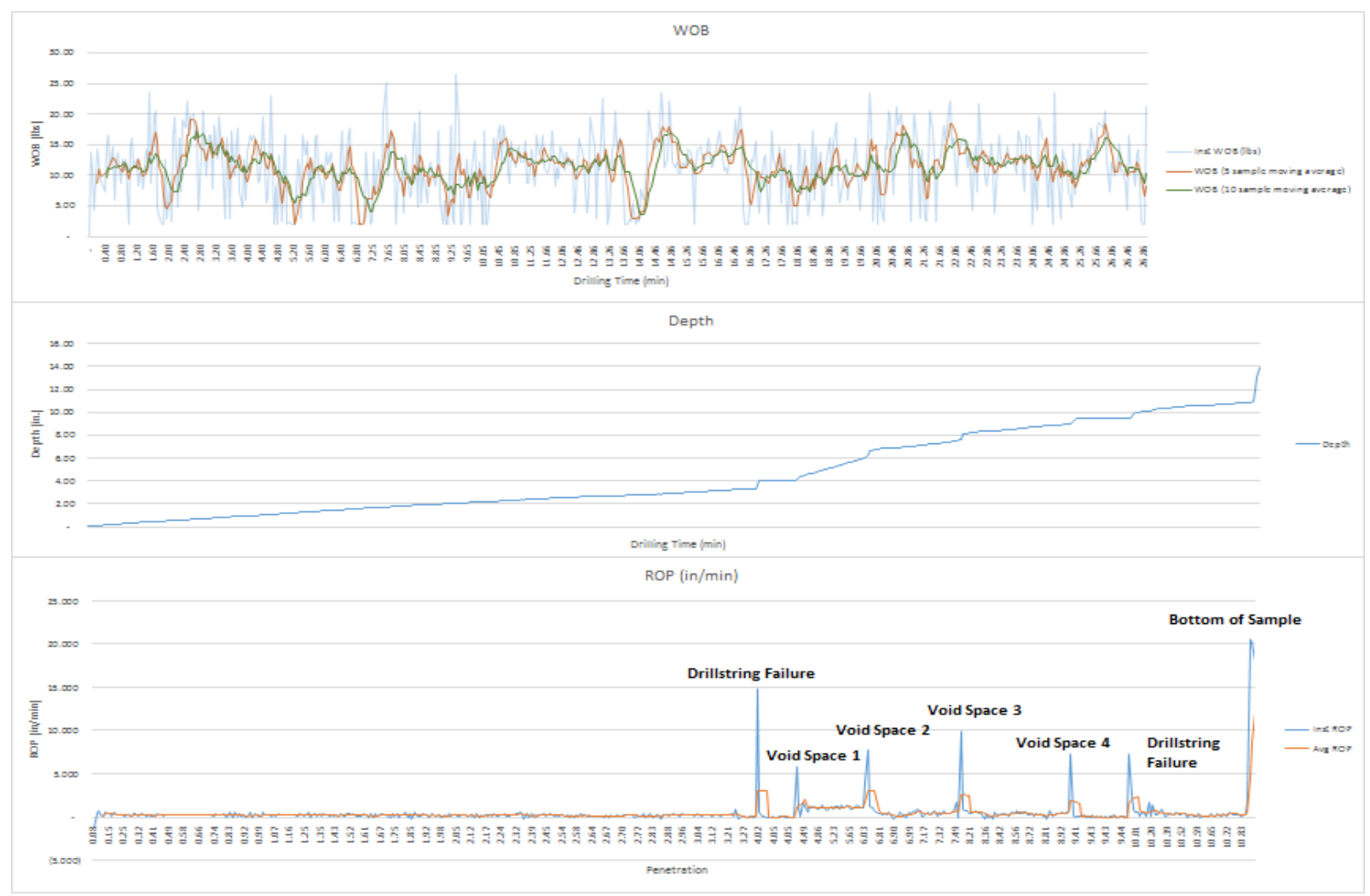

Figure 9 Competition Results

The depth reached provides insight into the overall function of the algorithm as it progressed. The first linear portion is quite long and shows no appreciable change in slope until the first drill string failure became insurmountable. This relatively low ROP is in some part due to the intermittent failure of the drill sting connector and the nature of the program itself. Due to the program using a stable ROP as the condition for repeating each drill off test, a constant ROP (constant slope) will keep the program from reiterating. It can be suspected that all drilling prior to the first drill string failure could have proceeded much faster as is evident in the latter portions drilled.

Analysis of the RPM-Torque set points showed that a total of nine optimized settings were selected for the five solid layers (with four void spaces) drilled. The RPM Torque graph is shown in Figure 10. It is interesting to note that the maximum RPM setting possible was 1750 and 
maximum torque possible was approximately $26 \mathrm{ft}$. lbs. This shows that the program did not aggressively select maximum values, but rather optimized ROP without direct selection of maximum parameter values. This is further verified in Figure 11, which shows that the power consumption never approaches the system maximum of $2.5 \mathrm{hp}$. In this case, the argument for energy efficient use is supported as additional power is not used to force a higher ROP.

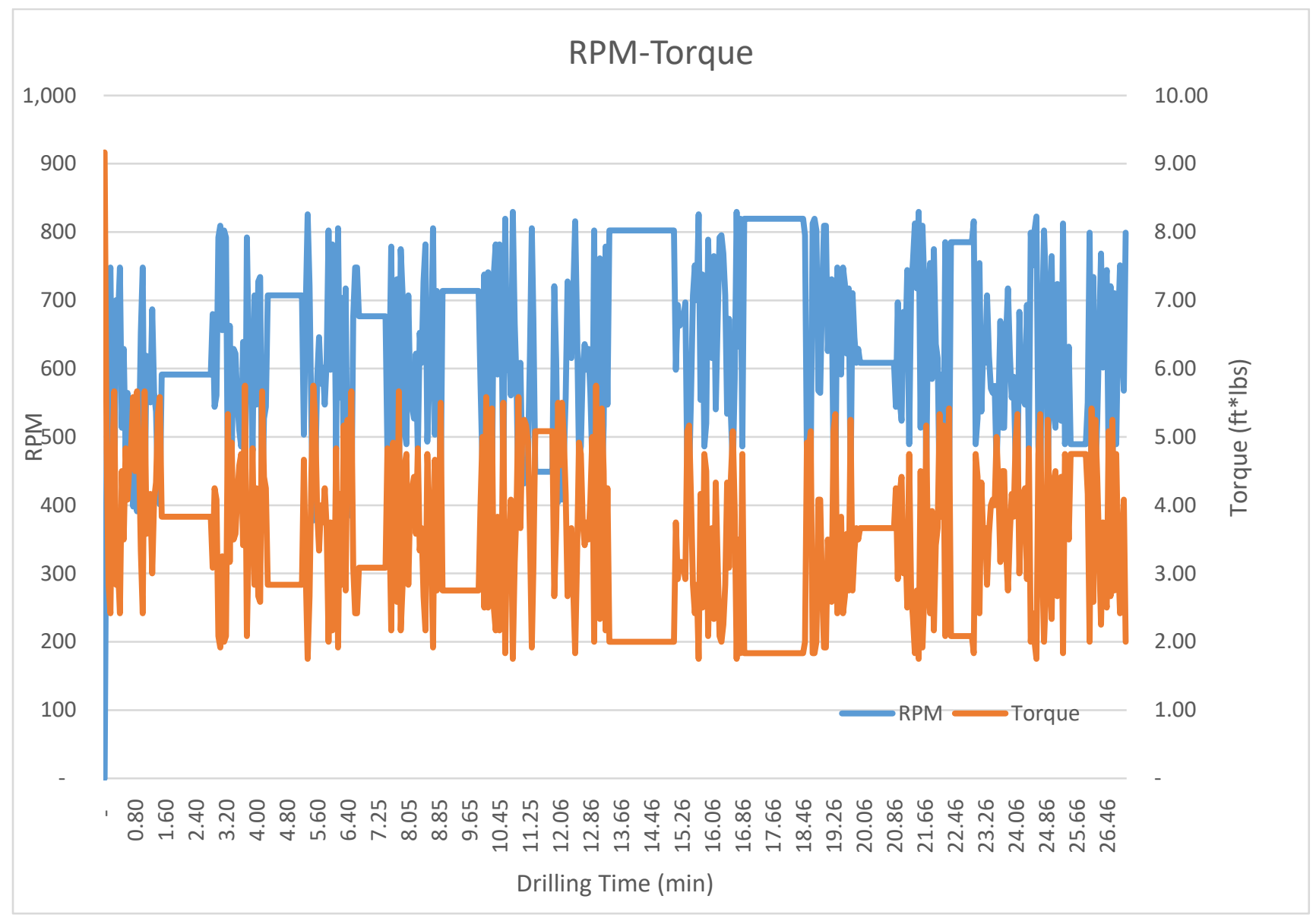

Figure 10 Recorded RPM-Torque 


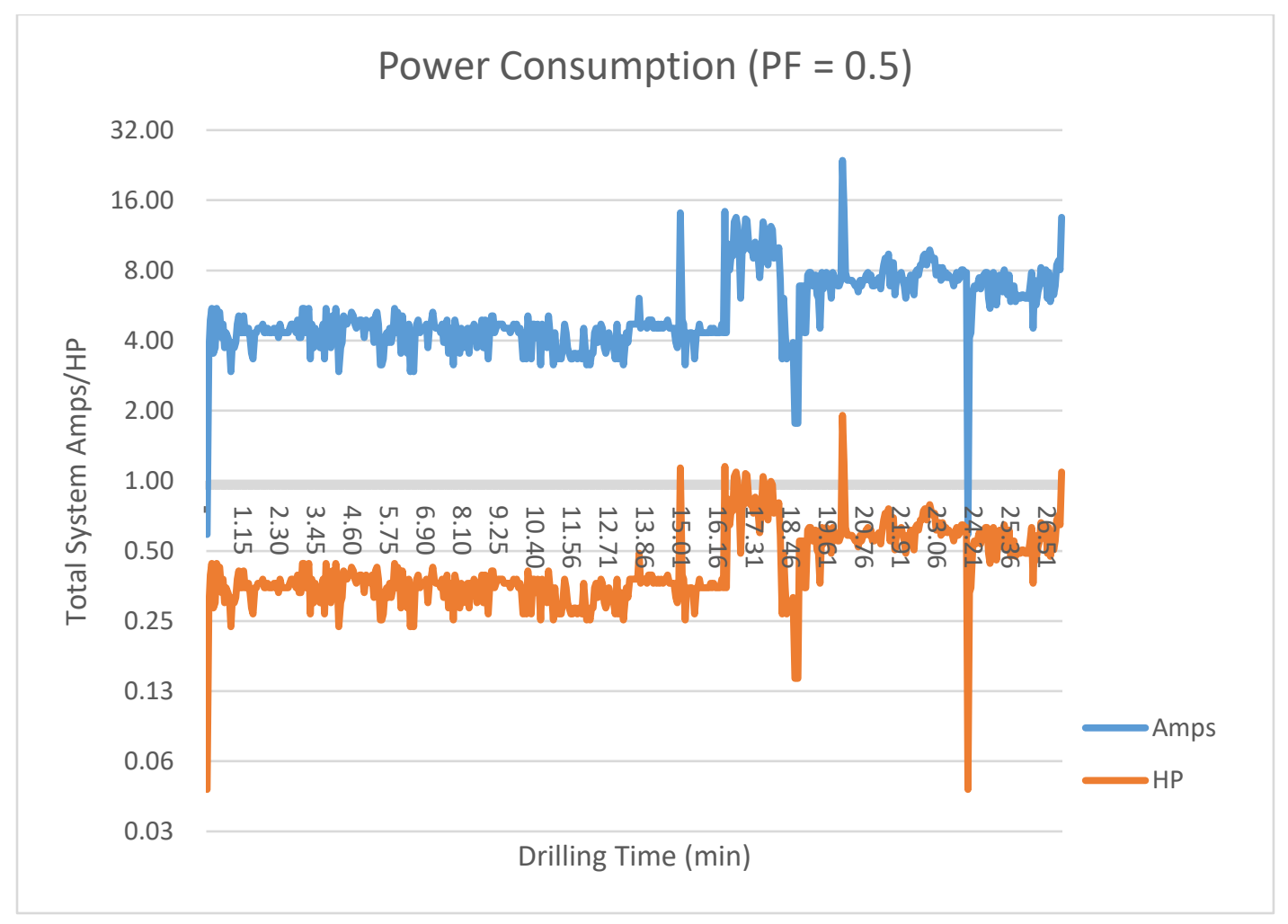

Figure 11 Recorded System Power Consumption

The average results are shown in Table 1. All values are significantly below the maximum possible allowed by the hardware. Average power consumed was $0.45 \mathrm{HP}$, significantly lower than either the drill or pump motor rating. This data suggests that lower power components could have been used while still maintaining optimal drilling parameters.

Table 1 Average Results

\begin{tabular}{|l|l|l|l|l|l|l|l|l|l|}
\hline $\begin{array}{l}\text { Drilling } \\
\text { time, min }\end{array}$ & $\begin{array}{l}\text { Distance } \\
\text { drilled, in }\end{array}$ & $\begin{array}{l}\text { WOB1 } \\
\text { bs }\end{array}$ & RPM & $\begin{array}{l}\text { ROP } \\
\text { in/min }\end{array}$ & $\begin{array}{l}\text { Torque } \\
\text { ft. }{ }^{*} \text { lbs. }\end{array}$ & $\begin{array}{l}\text { MSE, } \\
\text { psi }\end{array}$ & $\begin{array}{l}\text { Power } \\
\text { Consumption } \\
\text { HP }\end{array}$ & $\begin{array}{l}\text { Pump } \\
\text { pressure, } \\
\text { psi }\end{array}$ & $\begin{array}{l}\text { Flow } \\
\text { rate, } \\
\text { gpm }\end{array}$ \\
\hline 26.81 & 10.95 & 11.3 & 644 & 0.41 & 3.38 & 27,428 & 0.45 & 36 & 2.6 \\
\hline
\end{tabular}




\subsection{Remarks on Performance and Testing}

Performance of the rig while testing was overall acceptable and showed the promise of this technique for future applications. However, there is clearly room for additional improvements to be made to improve this technique and alter it for use in real-world applications. This would primarily cover additional implementations in the code to allow for more adaptability in the face of difficult downhole parameters. Testing also showed the effects of vibration, which were much greater on the small-scale rig than would be expected in large scale operations.

The performance of the algorithm adapted to changing ROP was evident. This was highlighted when the system encountered mechanical problems that the code could not anticipate or measure in any meaningful way. This provides adequate proof of concept for implementation of artificial intelligence algorithms in nontraditional environments and their application specifically to drilling. 


\section{Conclusions}

Based on the study, the following are presented:

- Rate of penetration optimization is a variable strategy for drilling an accurate, straight, and time efficient borehole.

- Optimizing ROP has been shown to improve the quality of the hole and the speed at which depth is reached. Furthermore, this allows for the reduction of vibrations which would otherwise hinder the quality of the wellbore.

- To a lesser extent, variation in optimized ROP also acts as an indicator layer homogeny. This effectively allows for the ROP to determine if the material being drilled is changing or otherwise different. This is important as it allows for a single parameter that can act to classify, indicate, and be optimized for drilling efficiency.

- The results of the study support the use of simulated annealing to select optimal parameters for enhance rate of penetration operation.

- While the simulated annealing algorithm must be adapted for use based on the constraints of any individual platform, it offers a simplistic way to allow for parameter selection. In this vain, the algorithm has shown to be applicable for the specific conditions of operation in this study.

- It is expected that with further modification, this method could be improved upon for a more complete and efficient algorithm to support more varied drilling operations.

- The methods employed in this study can apply to any drilling situation regardless of the material, bit type, and surrounding conditions. However, due to this wide applicability it should be noted that specific operations should generally outperform 
this method provided they are tailored to the specific circumstances. The algorithm and implementation supplied here is sufficient only when information about the drilling strata is minimal and the formations generally unknown. 


\section{Recommendations}

The work done here can be improved in three ways that are of interest to the problem presented for this study. These improvements can be summarized as multivariable optimization,

fault detection protocols, and hardware improvements. In addition, there are several incremental improvements that could be made to improve the human-machine interface. In practical applications, inclusion of manual control and interrupts are essential and would serve only to improve this test rig.

Multivariable optimization would allow for the speed at which the hole is drilled to be weighted with other variables to allow for simultaneous achievement of multiple goals. For instance, efficiency is not directly determined by the rate of penetration and could be used alongside it to provide for a lower power procedure. On the other hand, the bit wear induced by the drilling process could be accounted for and used to extend the life of the bit used. This could also be used to assist the program in optimizing variables by preselecting the ranges to be used for different bit types. Multivariable optimization would effectively create an entirely new version of this protocol and can be considered a significant and logical step forward in improving this method.

Fault detection protocols are secondary, and would offer additional practicality to the program and bridge the gap between small-scale and field-scale drilling. Fault detection is necessary prior to widespread adoption of this technology to ensure the safety not only of the workers onsite, but also the environment. This is because of the consequences of drilling through a fault, such as a broken pipe. This could cause leakage that would spill out and damage ether surrounding area. 
Lastly, an improved interface is the final consideration of any automation to be introduced into the workplace. This includes not only the application parameters while running but also the graphical user interface. The graphical user interface (GUI) is the only connection between a supervising operator and the functioning program that can alert them to dangers or allow them to control the algorithm without a hard connection. As such it is imperative that this be refined for broader use and include several operations that can be called for by an experience operator.

While automation is the key to moving forward it should be mentioned that any automations requires oversight. This brings with it the question of human interface and the extent that an operator can influence the program. Thus, it must also be designed around the human interaction that is anticipated to allow for optimal control and safety. 


\section{Bibliography}

1. Michalewicz, Z., \& Fogel, D. B. (2010). How to solve it: modern heuristics. Berlin: Springer.

2. Bavadiya, V. A., Aljubran, M. J., Kibe, J. M., Christy, S. M., Le, H. N., Ahmed, R., \& Florence, F. (2015). Design, Construction and Operation of an Automated Drilling Rig for the DSATS University Competition. SPE Annual Technical Conference and Exhibition. doi:10.2118/174920-ms

3. Breyholtz, O. \& Nikolaou, M. (2012). Drilling Automation: Presenting a Framework for Automated Operations. SPE Drilling \& Completion,27(01), 118-126. doi:10.2118/158109-pa

4. Cayeux, E., Daireaux, B., Dvergsnes, E., Leulseged, A., Bruun, B., \& Herbert, M. (2012). Advanced Drilling Simulation Environment for Testing New Drilling Automation Techniques and Practices. SPE Drilling \& Completion,27(04), 559-573. doi:10.2118/150941-pa

5. Macpherson, J. D., Wardt, J. P., Florence, F., Chapman, C., Zamora, M., Laing, M., \& Iversen, F. (2013). Drilling-Systems Automation: Current State, Initiatives, and Potential Impact. SPE Drilling \& Completion,28(04), 296-308. doi:10.2118/166263-pa 6. Thorogood, J. L. (2012). Automation in Drilling: Future Evolution and Lessons from Aviation. IADC/SPE Drilling Conference and Exhibition. doi:10.2118/151257-ms

7. Simpson, B. (1992). Field Trials of the First RA-D (Rig Automation-Drilling) Automated Drilling Rig. SPE/IADC Drilling Conference. doi:10.2118/23894-ms 8. Parr, E. A. (2000). Industrial control handbook. Oxford: Newnes 
9. Admin. (2017, March 17). The Intel ${ }^{\circledR}$ Edison Module | IoT. Retrieved December 17, 2016, from http://www.intel.com/content/www/us/en/do-it-yourself/edison.html 10. Arduino Forum. (n.d.). Retrieved February 21, 2017, from http://forum.arduino.cc/

11. The C Resources Network. (n.d.). Retrieved February 21, 2017, from http://www.cplusplus.com/

12. How Automated Optimization Can Push the Limits of ... (n.d.). Retrieved March 9, 2017, from https://www.pumpsandsystems.com\%2fpumps\%2fmay-2015-howautomated-optimization-can-push-limits-efficiency\&p=DevEx,5061.1

13. Roshan, S. B., Jooibari, M. B., Teimouri, R., Asgharzadeh-Ahmadi, G., FalahatiNaghibi, M., \& Sohrabpoor, H. (2013). Optimization of friction stir welding process of AA7075 aluminum alloy to achieve desirable mechanical properties using ANFIS models and simulated annealing algorithm. The International Journal of Advanced Manufacturing Technology,69(5-8), 1803-1818. doi:10.1007/s00170-013-5131-6 14. Hamrick, T. (2011). Optimization of Operating Parameters for Minimum Mechanical Specific Energy in Drilling. doi:10.2172/1060223

15. Teale, R. (1965). The concept of specific energy in rock drilling. International Journal of Rock Mechanics and Mining Sciences \& Geomechanics Abstracts,2(2), 245. doi:10.1016/0148-9062(65)90016-1 